\title{
Immuno-PET of epithelial ovarian cancer: harnessing the potential of CA125 for non-invasive imaging
}

Sai Kiran Sharma ${ }^{1,2}$, Melinda Wuest ${ }^{2}$, Monica Wang ${ }^{2}$, Darryl Glubrecht ${ }^{2}$, Bonnie Andrais ${ }^{2}$, Suzanne E Lapi ${ }^{3}$ and Frank Wuest ${ }^{1,2^{*}}$

\begin{abstract}
Background: Epithelial ovarian cancer (EOC) is characterized by the overexpression of cancer antigen 125 (CA125), a mucinous glycoprotein that serves as a tumor biomarker. Early diagnosis of EOC is plagued by its asymptomatic nature of progression and the limitations of currently used immunoassay techniques that detect CA125 as a shed antigen in serum samples. Presently, there is no technique available for the in vivo evaluation of CA125 expression in malignant tissues. Moreover, there could be an unexplored pathophysiological time window for the detection of CA125 in EOC, during which it is expressed on tumor cells prior to being shed into the bloodstream. A method for the in vivo evaluation of CA125 expression on ovarian neoplasms earlier along disease progression and/or recurrence can potentially contribute to better disease management. To this end, the present work utilizes an anti-CA125 monoclonal antibody (MAb) and a single-chain variable fragment (scFv) labeled with the positron-emitting radionuclide ${ }^{64} \mathrm{Cu}$ for preclinical molecular imaging of CA125 expression in vivo.
\end{abstract}

Methods: Anti-CA125 MAb and scFv were prepared and functionally characterized for target binding prior to being tested as radiotracers in a preclinical setting.

Results: Immunoblotting, immunofluorescence, and flow cytometry revealed specific binding of CA125-targeting vectors to $\mathrm{NIH}$ :OVCAR-3 cells and no binding to antigen-negative SKOV3 cells. ${ }^{64} \mathrm{Cu}$-labeled anti-CA125 MAb and scFv were obtained in specific activities of 296 and $122 \mathrm{MBq} / \mathrm{mg}$, respectively. Both radioimmunoconjugate vectors demonstrated highly selective binding to NIH:OVCAR-3 cells and virtually no binding to SKOV3 cells. In vivo radiopharmacological evaluation using xenograft mouse models injected with ${ }^{64} \mathrm{Cu}$-labeled anti-CA125 MAb provided a standardized uptake value (SUV) of $5.76(29.70 \% \mathrm{ID} / \mathrm{g}$ ) in OVCAR3 tumors $24 \mathrm{~h}$ post-injection (p.i.) versus $1.80(5.91 \% \mathrm{ID} / \mathrm{g})$ in SKOV3 tumors. ${ }^{64} \mathrm{Cu}$-labeled anti-CA125 scFv provided an SUV of $0.64(3.21 \% \mathrm{ID} / \mathrm{g})$ in OVCAR3 tumors $24 \mathrm{~h}$ p.i. versus $0.25(1.49 \% \mathrm{ID} / \mathrm{g})$ in SKOV3 tumors. Results from small-animal PET imaging were confirmed by ex vivo autoradiography and immunohistochemistry.

Conclusions: Radiolabeling of anti-CA125 MAb and scFv with ${ }^{64} \mathrm{Cu}$ did not compromise their immunoreactivity. Both radioimmunoconjugates presented specific tumor uptake and expected biological clearance profiles. This renders them as potential immuno-PET probes for targeted in vivo molecular imaging of CA125 in EOC.

Keywords: Antibody; CA125; Copper-64; Positron emission tomography; Epithelial ovarian cancer

\footnotetext{
* Correspondence: wuest@ualberta.ca

${ }^{1}$ Faculty of Pharmacy and Pharmaceutical Sciences, University of Alberta,

8613 - 114 Street, Edmonton, AB T6G 2H1, Canada

${ }^{2}$ Department of Oncology, University of Alberta, 11560 University Avenue,

Edmonton, AB T6G 1Z2, Canada

Full list of author information is available at the end of the article
} 


\section{Background}

Despite its relative rarity in the general population and the availability of standard treatment through surgical intervention and platinum chemotherapy, epithelial ovarian cancer (EOC) continues to be the most insidious and lethal gynecologic malignancy in the western world. The Surveillance Epidemiology and End Results (SEER) program has estimated 21,980 new cases of ovarian cancer to occur in 2014, with 14,270 deaths resulting from this disease in the United States alone [1]. A combination of the asymptomatic nature of this malignancy and the high incidence of recurrence results in a low 5-year survival rate of $44.6 \%$ [1].

Cancer antigen 125 (CA125) is a high molecular weight mucinous glycoprotein overexpressed on the membrane of EOC cells [2-4]. It has clinical relevance as a standardof-care serum biomarker for ovarian cancer surveillance despite expression in some non-gynecologic malignancies and benign conditions such as pregnancy, menstruation, endometriosis, liver disease, and congestive heart failure $[3,4]$. Post-treatment elevation of serum CA125 level in EOC patients serves as an indicator of progressive disease and finds clinical application in the management of patients with documented evidence of ovarian cancer [3-7]. However, a normal CA125 serum titer does not necessarily imply an absence of the disease. This is exemplified by the fact that an estimated $50 \%$ of patients with normal levels of CA125 post-chemotherapy have small volumes of active disease at second-look surgery, and a large portion of this population succumbs to recurrence from the disease [5,6]. Presently, serial CA125 levels in patients are evaluated using immunoassays.

However, despite their indicative utility, such assays can be far from delivering a true representation of the in vivo pathophysiological pattern and may be limited in their capabilities for early detection of ovarian neoplasms.

On a time scale of events for the diagnosis of recurrent ovarian cancer, it is known that radiological detection of the disease precedes the presentation of clinically diagnosable symptoms by 2 to 3 months. Furthermore, there is a median lag of 2 months between the elevation of CA125 alone and the radiological detection of this disease [5,6]. Among contemporary imaging modalities, $\left[{ }^{18} \mathrm{~F}\right]$ FDG-PET has shown the highest sensitivity and accuracy in detecting recurrent ovarian cancer, while simultaneous investigations with CT, MRI, and ultrasound which primarily detect morphological features and anatomical changes that can be distorted on account of primary cyto-reductive surgery in patients yielded negative or equivocal results [8-11].

Considering these facts, we hypothesized that a noninvasive approach using targeted immuno-PET to directly image the in vivo overexpression of CA125 on epithelial ovarian neoplasms may detect the malignancy at an earlier time, while providing a more accurate in vivo evaluation of tumor load and residual disease. Further, this approach could gain relevance when used in a pathophysiological time window wherein CA125 expression is limited to the surface of ovarian neoplasms prior to its shedding into the bloodstream. Our hypothesis is strengthened further by a mathematical model proposed by Hori and Gambhir [12] whereby an ovarian tumor may grow for 10.6 years before attaining a size of $10.52 \mathrm{~mm}^{3}$ before it starts to shed just enough CA125 antigen $(1.5 \mathrm{U} / \mathrm{mL})$ detectable by immunoassay methods used in the clinic.

Owing to its ability for targeting CA125 in ovarian cancer, MAb-B43.13 has been employed as an immunotherapeutic agent in the treatment of EOC [13-15]. More recently, immuno-PET has emerged as a strategy that unifies the specificity of antibody-based targeting with the sensitivity for detection imparted by positron-emitting radionuclides in PET in order to render a superlative diagnostic potential [16].

Findings from previous research with MAb-B43.13 and more recent reports for antibody-based radiotracers targeting biomarkers such as PSMA [17] and CA19-9 [18], which have similar pathophysiological nature in prostate and pancreatic cancers, respectively, motivated us to develop an immuno-PET strategy for the in vivo imaging of EOC. Additionally, we developed an antibody fragment of MAb-B43.13, in order to produce a radiotracer that could retain antigen-binding properties like the full-length antibody but achieve faster in vivo clearance and better tumor penetration as a function of its smaller molecular size, which may ultimately yield high-contrast in vivo PET images at earlier time points post-injection. To this end, MAb-B43.13 and its derivative single-chain variable fragment (scFv-B43.13) were labeled with positron emitter ${ }^{64} \mathrm{Cu}\left(t_{1 / 2} 12.7 \mathrm{~h}\right)$ and analyzed in vitro and in vivo using CA125 overexpressing NIH:OVCAR-3 cells and CA125negative SKOV3 cells in preclinical EOC xenograft mouse models.

\section{Methods}

\section{Expression and purification of anti-CA125 MAb and scFv}

The murine monoclonal IgG1 antibody targeting human CA125 was produced from hybridoma B43.13 [19] that was kindly provided by Quest Pharma Tech Inc., Edmonton, Canada. The hybridoma cell culture supernatant was used to purify the MAb-B43.13 by protein G affinity (P-7700, Sigma-Aldrich, St. Louis, MO, USA) on a BioLogic DuoFlow ${ }^{\mathrm{TM}}$ chromatography system (7600135, Bio-Rad Laboratories, Inc., Hercules, CA, USA). MAb-B43.13-derived anti-CA125 scFv was produced with modifications to previously described constructs [20,21]. Briefly, the DNA sequence encoding scFv-B43.13 was 
cloned into a pET22b + vector with a modified inter-chain linker, and the protein was expressed in Escherichia coli Rosetta 2 DE3 (Novagen, 71400-3, Merck KGaA, Darmstadt, Germany). The C-terminal hexa-histidinetagged $\mathrm{scFv}$ was purified from the soluble fraction of recombinant cell lysates by immobilized metal affinity chromatography using a TALON ${ }^{\circledR}$ Superflow resin (635507, Clontech Laboratories, Inc., Mountain View, CA, USA).

\section{Cell lines and culture conditions}

Ovarian cancer cell lines NIH:OVCAR-3 (ATCC ${ }^{\bullet}$ HTB$161^{\mathrm{Tm}}$, ATCC, Manassas, VA, USA) and SKOV3 (ATCC ${ }^{\oplus}$ HTB- $77^{\mathrm{TN}}$ ) were used for in vitro and in vivo studies. Cells were cultured in DMEM-F12 medium supplemented with $10 \% v / v$ fetal bovine serum (FBS), $50 \mathrm{IU} / \mathrm{mL}$ penicillin, and $50 \mu \mathrm{g} / \mathrm{mL}$ streptomycin (Gibco ${ }^{\circ}$, Life Technologies, Carlsbad, CA, USA). NIH:OVCAR-3 cells were additionally supplemented with $7 \mu \mathrm{g} / \mathrm{mL}$ recombinant human insulin (91077C, SAFC Biosciences, Inc., Lenexa, KS, USA). Cells were cultured using sterile techniques and grown in a $37^{\circ} \mathrm{C}$ incubator providing humidified atmosphere of $5 \%$ $\mathrm{CO}_{2}$ in air.

\section{Characterization of CA125-targeting vectors Western blotting}

NIH:OVCAR-3 and SKOV3 cells $\left(7.5 \times 10^{6}\right)$ were lysed with CelLytic $^{\mathrm{Tm}}$ M (C2978, Sigma-Aldrich). The cell lysates were electrophoresed on a $4 \%$ to $15 \%$ Mini-PROTEAN $^{\circledR}$ TGX $^{\mathrm{TM}}$ precast gel (456-1085, Bio-Rad) and transferred to a Trans-Blot nitrocellulose membrane (162-0115, BioRad). The membranes were probed separately to evaluate MAb versus $\mathrm{scFv}$ binding to the target antigen in cell lysates. The blots were blocked for $45 \mathrm{~min}$ with $5 \%$ non-fat dry milk (Carnation) in PBS having 0.1\% Tween-20 (PBST). Anti-CA125 MAb (3 mg/mL), mouse anti- $\beta$ actin IgG (A1978, Sigma-Aldrich), and anti-CA125 scFv (3 mg/ $\mathrm{mL}$ ) were used as primary antibodies to probe the blots at 1:5,000 dilutions for $1 \mathrm{~h}$ at room temperature. Goat anti-mouse HRP conjugate (A4416, Sigma-Aldrich) was used as secondary antibody to probe the blot against antiCA125 MAb and mouse anti- $\beta$ actin IgG at 1:5,000 dilution for $1 \mathrm{~h}$ at room temperature. 6xHis MAb-HRP conjugate (631210, Clontech) was used as secondary antibody at 1:5,000 dilution to probe against anti-CA125 $\mathrm{scFv}$ for $1 \mathrm{~h}$ at room temperature. The blots were washed with PBST and developed on Amersham Hyperfilm ECL (28906839, GE Healthcare, Little Chalfont, UK) using Amersham ECL Plus Western Blotting Detection Reagents (RPN2132, GE Healthcare).

\section{Fluorescent labeling of anti-CA125 MAb/scFv}

One milligram each of anti-CA125 MAb and scFv at concentrations of $2 \mathrm{mg} / \mathrm{mL}$ were fluorescently labeled using the Pierce $^{\bullet}$ fluorescein isothiocyanate (FITC) antibody labeling kit (53027, Thermo Scientific, Waltham, MA, USA) according to the manufacturer's instructions.

\section{Flow cytometry}

NIH:OVCAR-3 cells $\left(1.5 \times 10^{6}\right)$ were harvested by trypsinization, rinsed twice with fluorescence-activated cell sorting (FACS) buffer (PBS with $0.5 \%$ heat-inactivated FBS, $2 \mathrm{mM}$ EDTA, 0.05\% sodium azide), and resuspended by gentle tapping in an approximately $100 \mu \mathrm{l}$ FACS buffer. Ten micrograms of anti-CA125 MAb or $\mathrm{scFv}$ was incubated with the cell suspension for $30 \mathrm{~min}$ at room temperature. Cells were rinsed twice in FACS buffer and incubated for $30 \mathrm{~min}$ with $4 \mu \mathrm{g}$ of Alexa Fluor $^{\curvearrowleft} 488$ goat anti-mouse antibody (A-11001, Life Technologies) for the MAb samples and $4 \mu \mathrm{g}$ of Penta . His Alexa Fluor 488 conjugate (35310, Qiagen, Venlo, the Netherlands) for the scFv samples. Cells were rinsed twice with FACS buffer and analyzed by flow cytometry on a BD FACS Calibur (BD Biosciences, San Jose, CA, USA). A dengue virus targeting IgG1-12A1 and a hexahistidine-tagged anti-RANK receptor binding $\mathrm{scFv}$ were used as isotype controls for the experiments. Negative controls included unstained NIH:OVCAR-3 cells and cells incubated with Alexa Fluor 488 conjugated antibodies alone.

\section{Immunofluorescence}

NIH:OVCAR-3 and SKOV3 cells were plated onto glass coverslips in $35-\mathrm{mm}$ tissue culture dishes $(100,000$ cells/ $2 \mathrm{~mL}$ medium/dish) and incubated at $37^{\circ} \mathrm{C}$ for $48 \mathrm{~h}$. The cells were rinsed with PBS and fixed in methanol for $30 \mathrm{~min}$ at $-20^{\circ} \mathrm{C}$. The fixed cells were incubated in $5 \%$ non-fat dry milk (Carnation) in PBS for $30 \mathrm{~min}$ and immunostained separately for $1 \mathrm{~h}$ with FITC-labeled versus unlabeled anti-CA125 MAb and scFv. The unlabeled $\mathrm{MAb}$ and scFv samples were indirectly stained with corresponding Alexa Fluor 488-labeled secondary antibodies as used for flow cytometry experiments.

Anti-CA125 MAb and scFv (2 mg/mL) were used at 1:250 dilution followed by 1:500 dilutions of secondary antibodies $(2 \mathrm{mg} / \mathrm{mL})$ in PBS containing 5\% non-fat dry milk. Appropriate blank and control samples were included in the experiments. Antibody incubations were followed by three rinses with PBST for 5 min each. Coverslips were mounted on microscopy slides (Fisherbrand, 12-550-003, Thermo Fisher Scientific) using Mowiol $^{\circledR}$ mounting medium (Calbiochem, 475904, Millipore Co., Billerica, MA, USA) supplemented with DAPI (50 $\mu \mathrm{g} /$ $\mathrm{mL}$ ). Immunofluorescence was observed through a Zeiss Plan Apochromat 40X/1.3 Oil DIC M27 lens on a confocal laser scanning microscope (Zeiss LSM 710, Carl Zeiss AG, Oberkochen, Germany), and images were analyzed using Zen 2011 software. Separately, $30 \mu \mathrm{g}$ of FITClabeled anti-CA125 MAb was added to NIH:OVCAR-3 
cells grown on coverslips and allowed to incubate under standard cell culture conditions over a period of $48 \mathrm{~h}$ to study uptake in live cells. Coverslips were washed and mounted on glass slides for analysis by confocal microscopy at 0.5-, 1-, 4-, 12-, 24-, and 48-h time points.

\section{Surface plasmon resonance}

Kinetic constants for association $\left(k_{\mathrm{a}}\right)$ and dissociation $\left(k_{\mathrm{d}}\right)$ and affinity constant $K_{\mathrm{D}}$ for anti-CA125 MAb-B43.13 and $\mathrm{scFv}$-B43.13 were determined by surface plasmon resonance on a Biacore 3000 instrument (GE Life Sciences, Piscataway, NJ, USA). Upon activation of a CM5 sensor chip with a 1:1 mixture of $N$-hydroxysuccinimide/ $N$-ethyl$N^{\prime}$-(3-dimethylaminopropyl)-carbodiimide hydrochloride, amine coupling of antigen-grade human ovarian cancer native CA125 protein (MBS318371, MyBioSource, Inc., San Diego, CA, USA) dissolved in $10 \mathrm{mM}$ sodium acetate buffer $\mathrm{pH} 5.0$ was performed to couple approximately 1,200 relative units. The remaining reactive groups in the control and test lanes were inactivated using ethanolamine from the amine coupling kit (GE Life Sciences). The binding of antibody vectors to CA125 immobilized on the CM5 chip was assessed in duplicates across concentration ranges between $18.7 \mathrm{nM}$ and $9.33 \mu \mathrm{M}$ for MAb-B43.13 and $19.6 \mathrm{nM}$ and $19.6 \mu \mathrm{M}$ for the scFv-B43.13, respectively. Each sample of $\mathrm{MAb} / \mathrm{scFv}$ in the aforementioned concentrations in binding buffer (10 mM HEPES, $\mathrm{pH}$ 7.0) was injected for $3 \mathrm{~min}$ at a flow rate of $30 \mu \mathrm{L} / \mathrm{min}$ to allow binding with the antigen. The binding buffer was allowed to flow over the sensor chip for $15 \mathrm{~min}$ at a rate of $30 \mu \mathrm{L} /$ min to allow for the dissociation of bound $\mathrm{MAb} / \mathrm{scFv}$ from the antigen on the chip. Next, the regeneration buffer $(10 \mathrm{mM}$ HEPES pH 7.0 supplemented with $800 \mathrm{mM}$ $\mathrm{KCl}$ ) was passed over the chip surface to achieve dissociation of any remaining bound analyte, followed by $2 \mathrm{~min}$ for stabilization before the next injection. BIAcore control software 3.2 was used to analyze the data, and the best 1:1 Langmuir binding fit was used to derive kinetic constants.

\section{Preparation of CA125-targeting radioimmunoconjugates General}

All glassware was rinsed with ultra-pure $\mathrm{HCl}$ (Fisherbrand, A508-P500, Thermo Fisher Scientific). Trace metal basis ultra-pure chemicals for buffer preparations were purchased from Sigma-Aldrich. All buffer solutions were treated with biotechnology-grade Chelex 100 (143-2832, Bio-Rad).

\section{NOTA functionalization}

p-SCN-Bn-NOTA [S-2-(4-isothiocyanatobenzyl)-1,4,7triazacyclononane-1,4,7-triacetic acid] (B-605, Macrocyclics, Dallas, TX, USA) was conjugated to anti-CA125 $\mathrm{MAb}$ and scFv to serve as a bifunctional chelator for ${ }^{64} \mathrm{Cu}$ radiolabeling. Briefly, a $6 \mathrm{M}$ excess of p-SCN-Bn-NOTA in DMSO was added to anti-CA125 $\mathrm{MAb} / \mathrm{scFv}$ in $0.1 \mathrm{M}$ sodium bicarbonate buffer $\mathrm{pH} 9.0$ and allowed to react for $1 \mathrm{~h}$ at $37^{\circ} \mathrm{C}$. NOTA-functionalized anti-CA125 $\mathrm{MAb} / \mathrm{scFv}$ was purified from excess unconjugated bifunctional chelator while simultaneously achieving buffer exchange into $0.25 \mathrm{M}$ sodium acetate $\mathrm{pH} 5.5$ by using an Econo-Pac 10DG desalting column (732-2010, Bio-Rad). Protein quantification of the column-eluted fractions was performed using $\mathrm{A}_{280}$ measurements on nanodrop 2000 (Thermo Scientific) and bicinchoninic assay (Pierce BCA Protein Assay kit, 23227) using a Synergy $\mathrm{H} 1$ multimode microplate reader for $\mathrm{A}_{562}$ absorbance read-out. The number of bifunctional chelates conjugated per MAb and $\mathrm{scFv}$ was determined by matrix-assisted laser desorption-ionization time-of-flight (MALDI-TOF) MS analysis and according to the method described by Cooper et al. [22]. The purified immunoconjugates were used in radiolabeling experiments.

\section{${ }^{64} \mathrm{Cu}$ labeling of NOTA-functionalized MAb/scFv}

${ }^{64} \mathrm{Cu}$ was produced via a ${ }^{64} \mathrm{Ni}(\mathrm{p}, \mathrm{n}){ }^{64} \mathrm{Cu}$ nuclear reaction on a CS-15 biomedical cyclotron at Washington University, St. Louis, USA as previously described [23] and supplied as high specific activity ${ }^{64} \mathrm{CuCl}_{2}$ in $0.1 \mathrm{~N} \mathrm{HCl}$. Ammonium acetate $\left(0.1 \mathrm{M}\right.$; pH 5.5) was added to ${ }^{64} \mathrm{CuCl}_{2}$ to form ${ }^{64} \mathrm{Cu}$-acetate ${ }^{64} \mathrm{Cu}(\mathrm{OAc})_{2}$ solution. To $100 \mu \mathrm{g}$ of NOTAfunctionalized $\mathrm{MAb} / \mathrm{scFv}, 85 \mathrm{MBq}$ of ${ }^{64} \mathrm{Cu}(\mathrm{OAc})_{2}$ was added and allowed to react on a thermomixer at $30^{\circ} \mathrm{C}$, $550 \mathrm{rpm}$ for $1 \mathrm{~h}$. EDTA ( $1 \mathrm{mM})$ was added to quench the reaction over $10 \mathrm{~min} .{ }^{64} \mathrm{Cu}$-labeled $\mathrm{MAb} / \mathrm{scFv}$ radioimmunoconjugates were purified on an Econo-Pac 10DG desalting column pre-equilibrated with $0.25 \mathrm{M}$ sodium acetate, $\mathrm{pH} 5.5$ used as the eluant. Elution fractions $(350 \mu \mathrm{L})$ were collected from the column, and the radioactivity was measured using an Atomlab 400 dose calibrator (Biodex, Shirley, NY, USA). Fifteen microliters of each elution fraction was electrophoresed on a 12\% SDS-PAGE gel under non-reducing conditions and evaluated by autoradiography on a BAS-5000 phosphorimager (Fujifilm, Tokyo, Japan). Radiochemical yields and purity were determined by instant thin layer chromatography on ITLC-SG (SGI001, Varian, Inc., Palo Alto, CA, USA), using 10 mM EDTA pH 5.5 as the eluant. Fractions containing high specific activity radioimmunoconjugates were used for in vitro and in vivo radiopharmacological experiments. Monoclonal IgG1 antibody $12 \mathrm{~A} 1$ was modified into an immunoconjugate and radiolabeled with ${ }^{64} \mathrm{Cu}$ as described above to serve as a non-specific isotype control for in vivo experiments.

\section{Functional characterization of CA125-targeting radioimmunoconjugates Cell uptake studies}

NIH:OVCAR-3 and SKOV3 cells were seeded to obtain 250,000 cells per well in 12-well tissue culture plates. 
Prior to the uptake experiment, the growth medium was removed and cells were rinsed twice with PBS and incubated in Krebs buffer at $37^{\circ} \mathrm{C}$ for $1 \mathrm{~h}$. The radioimmunoconjugate $(100 \mathrm{KBq})$ was added to each well except those assigned to measure background activity alone. Cell uptake was terminated at 5, 10, 15, 30, 60, 90, and 120 min by adding ice-cold Krebs buffer and rinsing the wells twice to wash away any unbound radioimmunoconjugates prior to lysing the cells with RIPA buffer.

The cell lysates were transferred to scintillation vials and measured for radioactivity using a $\gamma$-counter $\left(\right.$ Wizard $^{20}$ 2480 Automatic Gamma Counter, PerkinElmer, Ontario, Canada). Protein levels were quantified using a Pierce ${ }^{\mathrm{Tm}}$ BCA protein assay kit according to the manufacturer's recommendations. Cell uptake levels were normalized to percentage of the total amount of radioactivity per milligram of protein (\% radioactivity/mg protein) and plotted as a function of time. All experiments were performed in triplicates.

Immunoreactivity of the radioimmunoconjugates was assessed by cell binding assays according to the method of Lindmo et al. [24].

\section{In vivo experiments}

Xenograft models All animal experiments were carried out according to guidelines of the Canadian Council on Animal Care (CCAC) and approved by the local animal care committee of the Cross Cancer Institute. Six-weekold BALB/c nude female mice were obtained from Charles River labs (Quebec, Canada). The animals were housed in ventilated cages and provided food and water ad libitum. NIH:OVCAR-3 tumors were induced on the left shoulder by two subcutaneous injections of $15 \times 10^{6}$ and $10 \times 10^{6}$ cells in a $300 \mu \mathrm{L}$ suspension of $1: 1$ mixture of PBS and Matrigel (354234, BD Biosciences). The second injection of cells was administered at the same site within 7 to 10 days. NIH:OVCAR-3 tumors grew for 6 to 8 weeks before reaching suitable tumor sizes of 150 to $200 \mathrm{~mm}^{3}$.

SKOV3 tumors were induced on the left shoulder by a single subcutaneous injection of $5 \times 10^{6}$ cells in PBS and were grown for about 2 to 3 weeks before achieving similar tumor sizes.

\section{Animal imaging}

Small-animal PET experiments were performed on a MicroPET $^{\circ}$ R4 or INVEON PET scanner (Siemens Preclinical Solutions, Knoxville, TN, USA). Mice were anesthetized by inhalation of isoflurane in $40 \%$ oxygen/ $60 \%$ nitrogen, $1 \mathrm{~L} / \mathrm{min}$, while maintaining body temperature at $37^{\circ} \mathrm{C}$ throughout the experiment; 6 to $10 \mathrm{MBq}$ of high specific activity radioimmunoconjugate (approximately 30 to $40 \mu \mathrm{g}$ ) in 150 to $200 \mu \mathrm{L}$ of $0.25 \mathrm{M}$ sodium acetate ( $\mathrm{pH} 5.5)$ was administered intravenously via a tail vein catheter. For blocking experiments, $1 \mathrm{mg}$ of unmodified anti-CA125 MAb was administered intraperitoneally $24 \mathrm{~h}$ prior to injection of the ${ }^{64} \mathrm{Cu}$-labeled radioimmunoconjugates. In separate animals, 8 to $10 \mathrm{MBq}$ of ${ }^{64} \mathrm{Cu}$-labeled 12A1 IgG1 was administered via tail vein injection to evaluate non-specific uptake. Whole-body PET data was acquired by performing static scans for each animal at $24 \mathrm{~h}$ post-injection (p.i.) and $48 \mathrm{~h}$ p.i. Data acquisition continued for $60 \mathrm{~min}$ in 3D list mode. The image files were reconstructed using the maximum a posteriori (MAP) algorithm. The image files were further processed using the ROVER v 2.0.51 software (ABX GmbH, Radeberg, Germany). Masks for defining 3D regions of interest (ROI) were set, and the ROIs were defined by thresholding. Standardized uptake values $[\mathrm{SUV}=($ activity $/ \mathrm{mL}$ tissue)/(injected activity/body weight), $\mathrm{mL} \mathrm{g}^{-1}$ ] were calculated for each ROI. Additionally, radioactivity uptake was also analyzed as percentage of injected dose per gram tissue $(\% \mathrm{ID} / \mathrm{g})$. Data are expressed as means \pm SEM from $n$ investigated animals.

\section{Ex vivo analyses}

An NIH:OVCAR-3 tumor-bearing mouse was injected with $4.55 \mathrm{MBq}$ of ${ }^{64} \mathrm{Cu}$-labeled anti-CA125 MAb via the tail vein. At 24 h p.i., the animal was euthanized by cervical dislocation. The tumor was submerged into OCT medium and flash frozen using a bath of dry ice-cooled methanol. Seven-micrometer slices were cut on a Leica CM 1850 cryostat (Leica Microsystems, Singapore).

\section{Autoradiography}

Tissue sections were placed into a BAS-Cassette (2325, Fujifilm) and exposed to a phosphor imaging plate (BAS-MS 2025, Fujifilm) for $15 \mathrm{~h}$ at room temperature. The images were developed on a BAS-5000 reader (Fuji Photo Film Co., Ltd.) and analyzed with Adaptive Image Deconvolution Algorithm (AIDA) Image Analyzer v.450 software.

\section{Immunofluorescence}

Upon thawing, tissue sections were fixed with formalin for $30 \mathrm{~min}$. The fixed sections were blocked overnight at $4^{\circ} \mathrm{C}$ using $0.12 \mathrm{mg} / \mathrm{mL}$ unconjugated goat anti-mouse Fab fragment (115-003-007, Jackson Immunoresearch, West Grove, PA, USA) in 0.5\% fish skin gelatin (G7765, Sigma-Aldrich) pH 7.4, supplemented with $0.1 \%$ Triton $\mathrm{X}-100$. The sections were rinsed $3 \times$ with Tris-buffered saline having $0.5 \%$ Tween 20 (TBST) for 5 min each. MAb-B43.13 was used as primary antibody at a dilution of 1:6,000 in Dako antibody diluent (S0809, Dako, Glostrup, Denmark) and allowed to incubate overnight at $4^{\circ} \mathrm{C}$. The sections were rinsed $3 \times$ with TBST and incubated with Alexa Fluor 488 goat anti-mouse antibody (A-11001, Life Technologies) used as secondary 
antibody at 1:400 dilution in Dako antibody diluent for $2 \mathrm{~h}$ at room temperature. After three washes with TBST, the sections were rinsed with water and counterstained using Hoechst H33342 $(2 \mu \mathrm{g} / \mathrm{mL})$ for $5 \mathrm{~min}$. The sections were rinsed and mounted under a coverslip using FluorSave (345789, Calbiochem). The slides were analyzed with a Zeiss Plan Apochromat 10X/0.45 na lens on a confocal laser scanning microscope (Zeiss LSM 710) using Z-stack for image acquisition. The images were registered using Zen 2011 software (Zeiss) and further processed with Adobe Photoshop CS6.

\section{Immunohistochemistry}

To analyze the samples for immunohistochemistry, the same procedures for treatment, fixation, blocking, and probing of tissue with primary antibody were followed. DakoEnVision $^{\mathrm{Tw}}+$ HRP-conjugated anti-mouse antibody was used as a secondary antibody, and no counterstain was employed. The sections were observed with a Zeiss Fluar 2.5X/0.12 na lens on an Axioskop 2 Plus microscope. Images were processed and analyzed on AxioVision 4.7 (Zeiss) and further processed with Adobe Photoshop CS6.

\section{Statistical analysis}

All data are expressed as means \pm SEM. Graphs were constructed using GraphPad Prism 4.0 (GraphPad Software). Where applicable, statistical differences were tested by unpaired Student's $t$ test and were considered significant for $p<0.05$.

\section{Results}

Isolation, characterization, and modification of CA125targeting vectors

CA125-targeting vectors were purified in yields of $6 \mathrm{mg} / \mathrm{L}$ for MAb-B43.13 and $0.7 \mathrm{mg} / \mathrm{L}$ for the $\mathrm{scFv}$ (Figure 1A). Analysis by Western blot (Figure 1B) and immunofluorescence staining (Figure 2A) ascertained the following: a) expression of CA125 in NIH:OVCAR-3 cells, b) absence of CA125 expression in SKOV3 cells, and c) binding of MAb and $\mathrm{scFv}$ to target antigen CA125. Isothiocyanate-based labeling of anti-CA125 MAb and scFv with fluorescein yielded $2 \mathrm{~mol}$ of FITC per mole of MAb and scFv. SPR analysis calculated an affinity constant of $2.56 \times 10^{-9} \mathrm{M}$ for MAb-B43.13 and $1.11 \times 10^{-8} \mathrm{M}$ for the $\mathrm{scFv}$, with comparable dissociation constants in the range of $10^{-4} \mathrm{~s}^{-1}$ (Additional file 1: Table S1).

Immunofluorescence staining and flow cytometry analysis with the FITC-labeled MAb and scFv demonstrated preserved immunoreactivity by binding to CA125 antigen expressed on the membrane of NIH:OVCAR-3 cells, and no binding was seen with SKOV3 cells (Figure 2A, $\mathrm{B}, \mathrm{C})$.

FITC-labeled anti-CA125 MAb remained membrane bound over a period of $48 \mathrm{~h}$ with minimal cellular internalization observed upon incubation with live NIH: OVCAR-3 cells in culture (Figure 3).

\section{Development and functional assessment of CA125- targeting radioimmunoconjugates}

MALDI-TOF analyses and isotopic dilution assays revealed an average of 2.4 NOTA molecules conjugated per MAb and 1.9 NOTA molecules conjugated per scFv
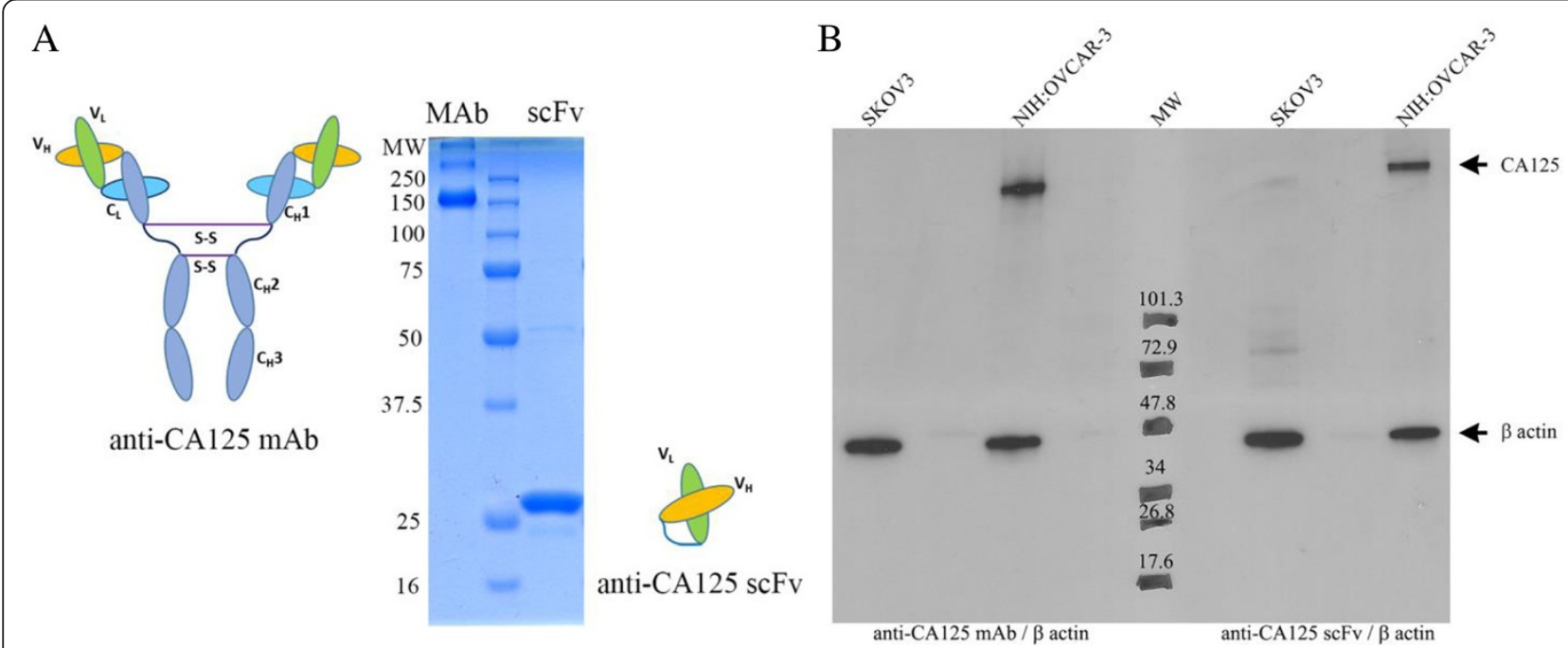

Figure 1 Representative images and Western blots. (A) Representative images of purified anti-CA125 MAb (148 KDa) and scFv (28 KDa) upon SDS-PAGE under non-reducing conditions. (B) Western blots of SKOV3 (CA125-) and NIH:OVCAR-3 (CA125+) cells with anti-CA125 MAb, anti- $\beta$ actin antibody (left side of molecular weight marker); anti-CA125 scFv, and anti- $\beta$ actin antibody (right side of molecular weight marker). 


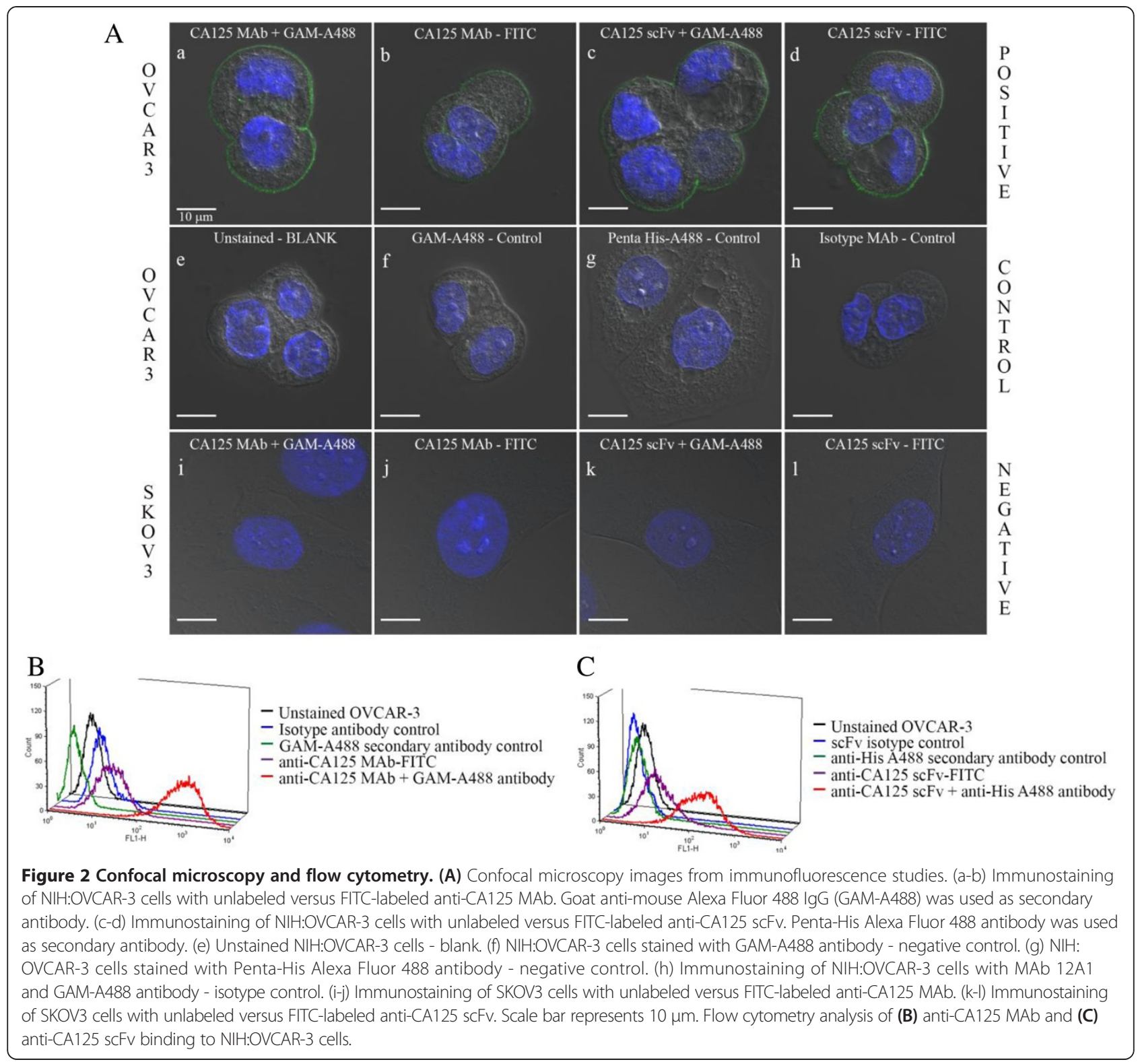

(Additional file 1: Figures S2 and S3). ${ }^{64} \mathrm{Cu}$-labeling and purification of radioimmunoconjugates provided isolated radiochemical yields of $65 \% \pm 8 \%(n=14)$ with specific activity of $296 \pm 37 \mathrm{MBq} / \mathrm{mg}$ for anti-CA125 MAb and $56 \% \pm 14 \%(n=9)$ with specific activity of $122 \pm$ $44 \mathrm{MBq} / \mathrm{mg}$ for anti-CA125 scFv (Figure 4).

Radiochemical purity of the isolated radioimmunoconjugates was greater than $99 \%$ as analyzed by ITLC (Additional file 1: Figure S4) and phosphor images of electrophoresed fractions (Additional file 1: Figure S5). Purified radioimmunoconjugates were $>95 \%$ stable in human AB-type serum over $48 \mathrm{~h}$ (Additional file 1: Figures $\mathrm{S} 6$ and $\mathrm{S} 7$ ) and demonstrated highly specific binding to CA125 expressed on NIH:OVCAR-3 cells with virtually no binding to SKOV3 cells as evaluated by cell uptake studies (Figure 5A, B). Immunoreactivity of ${ }^{64} \mathrm{Cu}$-labeled anti-CA125 MAb and scFv preparations was found to be $>90 \%$ as obtained from the inverse of the intercept of plots seen in Figure 5C, D, respectively.

\section{In vivo experiments and radiopharmacological evaluation}

PET imaging studies performed with ${ }^{64} \mathrm{Cu}$-labeled antiCA125 MAb and scFv in NIH:OVCAR-3 and SKOV-3 tumor-bearing mice as well as experiments with ${ }^{64} \mathrm{Cu}$ labeled isotype IgG and $\left[{ }^{18} \mathrm{~F}\right]$ FDG are summarized in Figure 6.

In NIH:OVCAR-3 tumors, ${ }^{64} \mathrm{Cu}$-labeled anti-CA125 MAb reached a maximum SUVmean of $5.76 \pm 0.85$ $(29.70 \pm 3.34 \% \mathrm{ID} / \mathrm{g})$ at $24 \mathrm{~h}$ p.i. rising to $7.06 \pm 0.67$ $(41.66 \pm 4.38 \% \mathrm{ID} / \mathrm{g})$ at $48 \mathrm{~h}$ p.i. $(n=4)$. NIH:OVCAR-3 

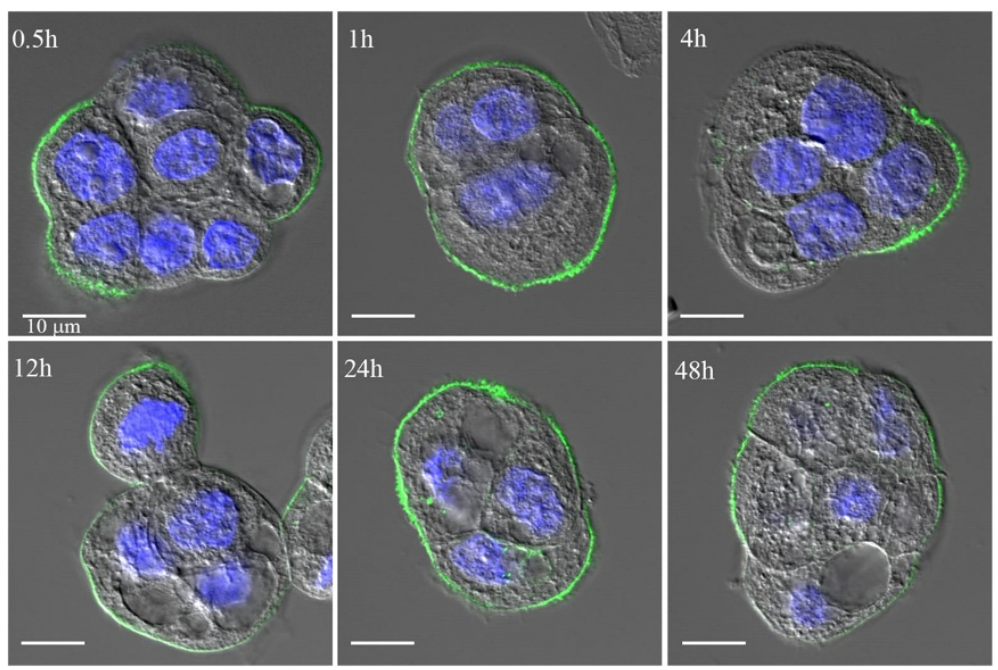

Figure 3 Confocal microscopy images of NIH:OVCAR-3 cells directly immunostained with FITC-labeled anti-CA125 MAb over 48 h.

xenograft mice pre-dosed with unlabeled MAb revealed a SUVmean of $2.90 \pm 0.45(13.10 \pm 2.07 \% \mathrm{ID} / \mathrm{g})$ at $24 \mathrm{~h}$ p.i. $(n=4, p<0.05)$ and $3.96 \pm 0.76(14.47 \pm 3.01 \% \mathrm{ID} / \mathrm{g})$ at 48 h p.i. $(n=4, p<0.05)$. Non-specific tumor uptake using ${ }^{64} \mathrm{Cu}$-labeled isotype IgG1 was found to have a SUVmean of $1.77 \pm 0.44(9.77 \pm 0.44 \% \mathrm{ID} / \mathrm{g})$ at 24 h p.i. $(n=3$, $p<0.05)$ with no further change at $48 \mathrm{~h}$ p.i. A SUVmean of $1.80 \pm 0.69(5.91 \pm 3.31 \% \mathrm{ID} / \mathrm{g}) 24 \mathrm{~h}$ p.i. $(n=5, p<0.01$ vs. uptake in OVCAR3 24 h p.i.) was observed in SKOV3 tumors.

In contrast, PET studies using ${ }^{64} \mathrm{Cu}$-labeled anti-CA125 $\mathrm{ScFv}$ in xenograft mice revealed a SUVmean of $0.64 \pm 0.04$ $(3.21 \pm 0.29 \% \mathrm{ID} / g) 24 \mathrm{~h}$ p.i. $(n=3)$ in NIH:OVCAR-3 tumors and $0.25 \pm 0.11(1.49 \pm 0.68 \% \mathrm{ID} / \mathrm{g}) \quad(n=3, p$ $<0.05$ ) in SKOV3 tumors.

Overall, in vivo clearance of the radioimmunoconjugates was evaluated from the heart (representing blood pool content), liver, and kidneys (Additional file 1: Figure S8).

After $24 \mathrm{~h}$, SUVmean in the heart and liver of xenograft animals imaged with ${ }^{64} \mathrm{Cu}$-labeled anti-CA125 MAb was found to be $2.40 \pm 0.20(12.61 \pm 1.15 \% \mathrm{ID} / \mathrm{g})$ and $2.59 \pm$ $0.20(12.54 \pm 1.67 \% \mathrm{ID} / \mathrm{g})(n=4)$, respectively. SUVmean in the kidneys of these animals was $1.64 \pm 0.09(10.35 \pm$ $1.06 \% \mathrm{ID} / \mathrm{g})$.

In contrast, injection of ${ }^{64} \mathrm{Cu}$-labeled anti-CA125 scFv led to a SUVmean $(n=3)$ of $0.31 \pm 0.06(1.55 \pm 0.40 \% \mathrm{ID} / \mathrm{g})$

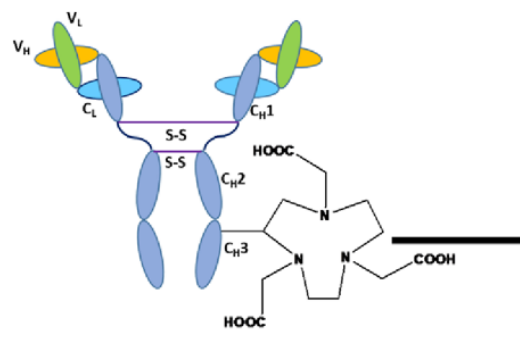

NOTA-conjugated anti-CA125 MAb

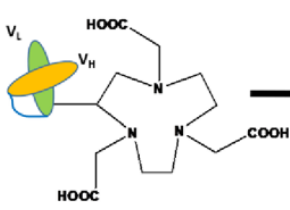

NOTA-conjugated anti-CA125 scFv

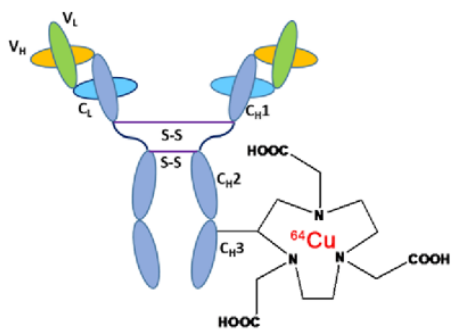

$\left[{ }^{64} \mathrm{Cu}\right]-$ Acetate

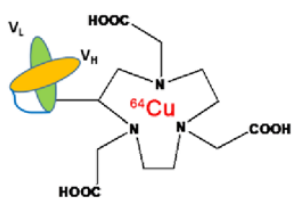

${ }^{64} \mathrm{Cu}-\mathrm{NOTA}$-anti-CA125 scFv

Figure 4 Schematic representation of ${ }^{64} \mathrm{Cu}$-labeling of NOTA conjugated anti-CA125 MAb and scFv. 


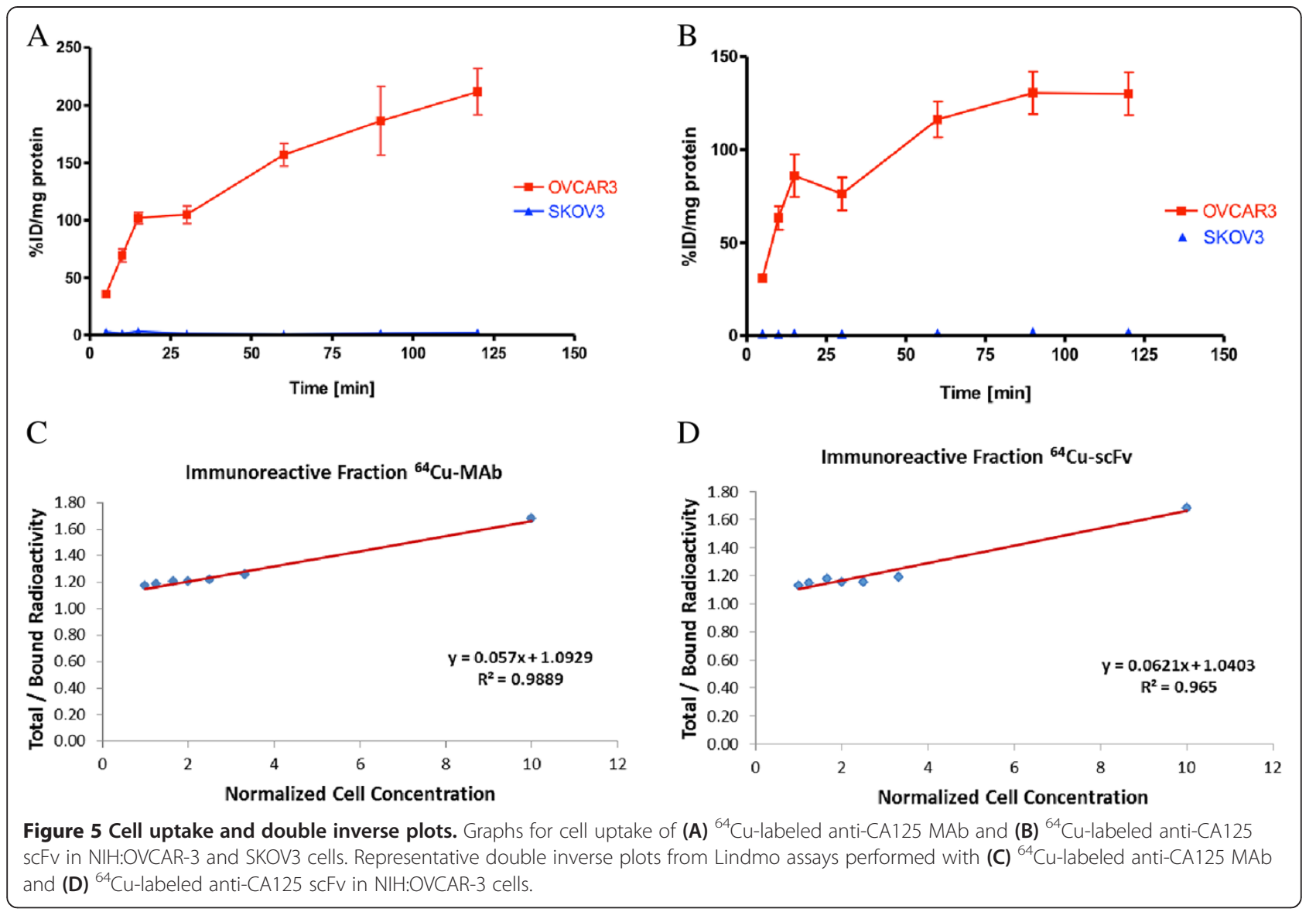

in the heart, $0.98 \pm 0.11(4.89 \pm 0.89 \% \mathrm{ID} / \mathrm{g})$ in the liver, and $13.2 \pm 1.2(66.46 \pm 9.20 \% \mathrm{ID} / \mathrm{g})$ in the kidneys after $24 \mathrm{~h}$. This information demonstrated the different in vivo clearance profile between the full-length antibody and the fragment.

\section{Ex vivo analysis}

The NIH:OVCAR-3 tumor tested positive for CA125 expression as seen from immunofluorescence and immunohistochemistry of tissue sections (Figure 7A, B, C, D, E, F).

Immunohistochemistry revealed necrotic and apoptotic regions spanned by connective tissue in the tumor's upper half. Overall, the lower half had more radioactive hot spots, stained stronger for CA125 expression, and had more vasculature surrounded by positively staining tumor foci, which collectively formed pockets of high signals. CA125 staining was observed on the surface of neoplastic cells in the tumor. Hot spots representative of in vivo targeting by the radiotracer as seen in autoradiography of tumor slices correlated well with signals from the other two techniques.

A good correlation of all three types of signals was observed in regions of tumor foci interspersed with blood vessels and/or supported by mouse connective tissue.

\section{Discussion}

EOC accounts for more than $90 \%$ of all ovarian carcinomas presented in the clinic, and $70 \%$ of these cases are high-grade serous ovarian cancers (HGSOC) overexpressing CA125 [25]. This offers a premise to target CA125 for in vivo diagnosis of ovarian neoplasms. Here, OVCAR3 human adenocarcinoma cells representative of HGSOCs and SKOV3 cells representative of human clear cell ovarian carcinoma that do not express CA125 were utilized in order to develop a robust preclinical testing platform to validate CA125 expression and targeting. Both the cell lines displayed consistent biochemical characteristics across in vitro, in vivo, and ex vivo analyses.

Unlike a previous report [26], we engineered MAbB43.13 using a standard bioconjugation strategy to radiolabel it with ${ }^{64} \mathrm{Cu}$ towards development of a CA125targeted radiotracer for immuno-PET. To the best of our knowledge, this is the first systematic report of an immuno-PET strategy for the in vivo targeting and imaging of CA125 in EOC. In contrast to OVCAR3 cells in culture, we found the uptake of $\left[{ }^{18} \mathrm{~F}\right]$ FDG in OVCAR3 tumors to be relatively low (Figure 6F), due plausibly to the cystic nature of ovarian tumors in vivo [27]. Thus, the utility of a targeted radiotracer that binds to cell surface 


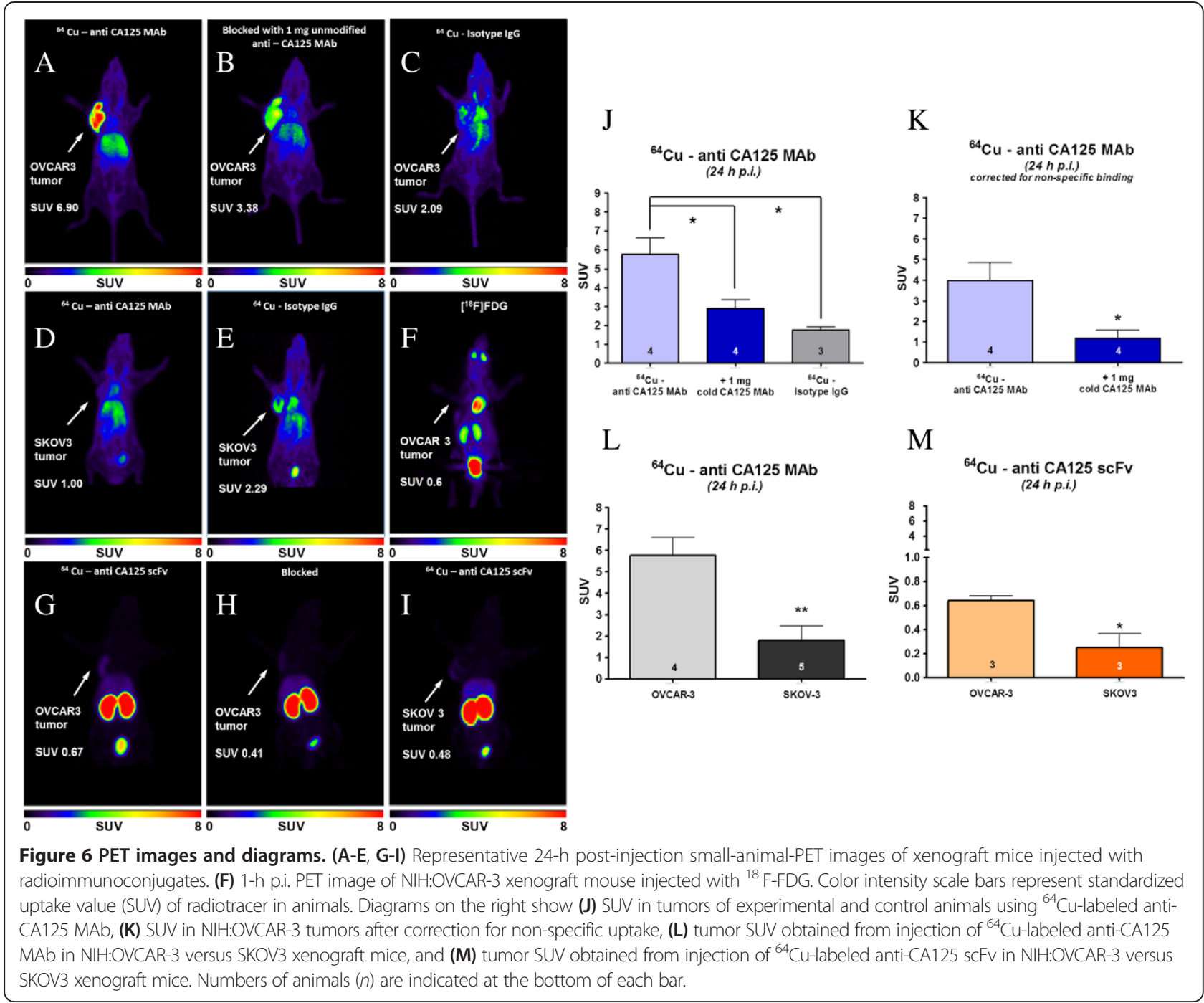

antigen may be more sensitive and efficient for the in vivo delineation of EOC.

In the present work, an isothiocyanate-based bioconjugation of a fluorescent tag and a macrocyclic chelator within prescribed molar ratios to the anti-CA125 MAb and $\mathrm{scFv}$ followed by radiolabeling yielded consistent results without compromising their immunoreactivity. This approach offers a scope for labeling MAb-B43.13based CA125-targeting vectors with different radionuclides, fluorophores, and functional groups for use in diverse imaging and therapeutic applications. Furthermore, based on indications from a previous report [28] and our own observations for non-internalization of surface antigen-bound MAb-B43.13 over $48 \mathrm{~h}$ as shown in Figure 3, pre-targeting applications are currently being explored via conjugation of click components to the anti-CA125 MAb-B43.13.

The present study with CA125-targeting MAb-B43.13 and its derivative $\mathrm{scFv}$ in preclinical ovarian cancer models found a higher targeted tumor uptake of ${ }^{64} \mathrm{Cu}$ NOTA-MAb-B43.13. Specificity of the observed in vivo tumor uptake was validated by pre-dosing OVCAR3 xenograft animals with $1 \mathrm{mg}$ of unmodified anti-CA125 $\mathrm{MAb} 24 \mathrm{~h}$ prior to injection of ${ }^{64} \mathrm{Cu}$-labeled anti$\mathrm{CA} 125 \mathrm{MAb}$ and scFv. The non-specific tumor uptake observed in our study was attributed to an enhanced permeability and retention effect (EPR) [29], whose numerical value (SUV) fell in range with the uptake of ${ }^{64} \mathrm{Cu}$-NOTA-MAb B43.13 observed in CA125-negative SKOV3 tumors.

The background radiation in all PET imaging studies using ${ }^{64} \mathrm{Cu}-\mathrm{NOTA}-\mathrm{MAb} \mathrm{B} 43.13$ could be attributed to a) its longer residency time in circulation, b) consistent non-specific muscle uptake of the radiotracer in all experimental animals (Additional file 1: Figure S9), c) hepatobiliary clearance of the radiotracer and its associated immunocomplexes, and d) potential in vivo transchelation of ${ }^{64} \mathrm{Cu}$ from NOTA by hepatic enzymes such 


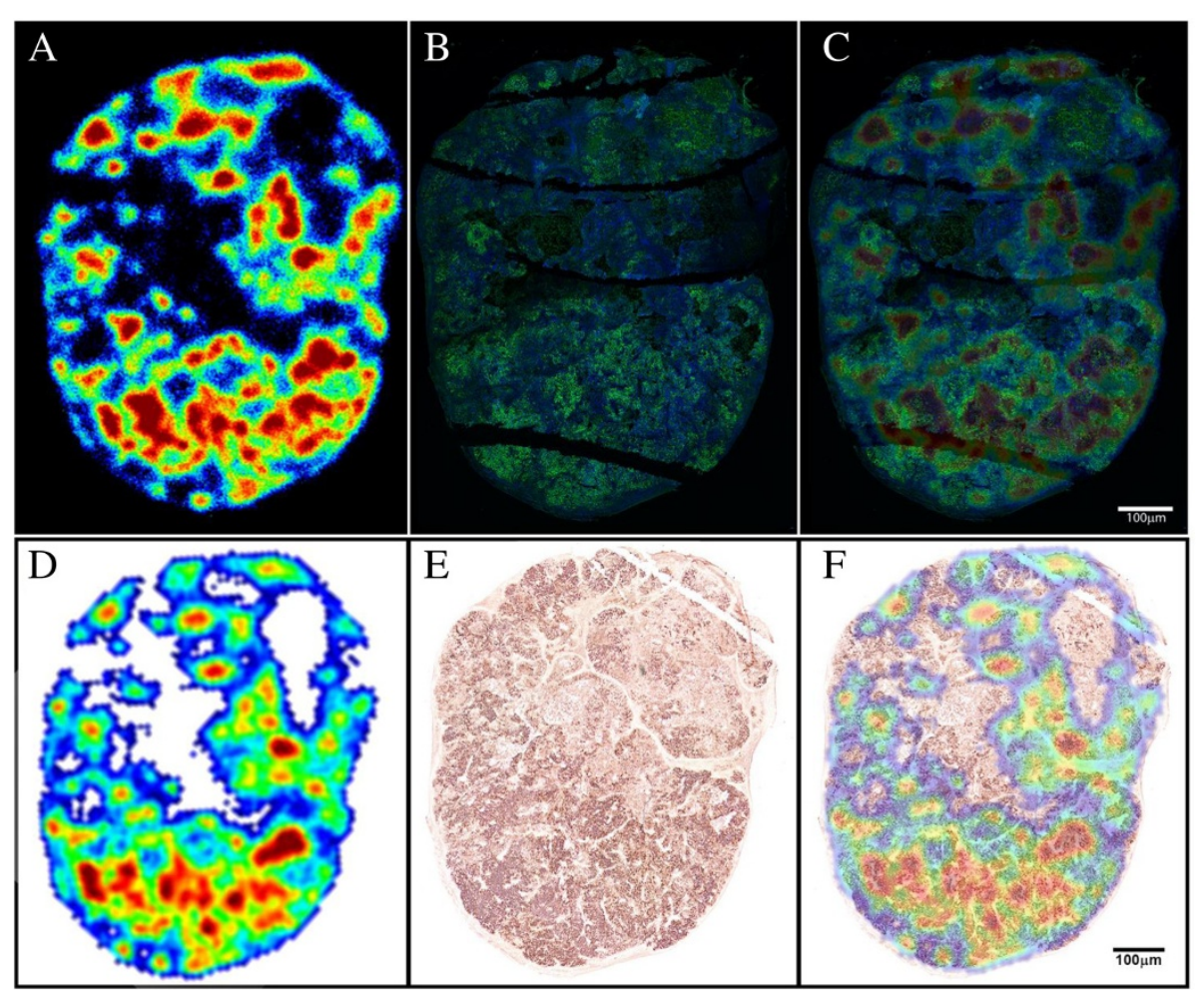

Figure 7 Autoradiography, immunofluorescence, immunohistochemistry, and overlay images. (A) Autoradiography image from a section of NIH:OVCAR-3 tumor indicating hot spots of in vivo targeting by ${ }^{64} \mathrm{Cu}$-labeled anti-CA125 MAb. (B) Immunofluorescence image of the same section staining green in regions of CA125 expression (Alexa Fluor 488) and counterstained blue (Hoechst 33342) for nuclei. (C) Overlay of images (A) and (B) to establish concurrence between signals from independent methods of ex vivo analysis to indicate CA125 expression and targeting. (D) Autoradiography image from a separate section of $\mathrm{NIH}$ :OVCAR-3 tumor indicating hot spots of in vivo targeting by ${ }^{64} \mathrm{Cu}$-labeled antiCA125 MAb. (E) Immunohistochemistry image of corresponding tissue section. Intensity of staining is indicative of CA125 expression across the sample. (F) Overlay of images (D) and (E) to establish concurrence between signals from independent methods of ex vivo analysis to indicate CA125 expression and targeting.

as superoxide dismutase (SOD). However, NOTA has previously demonstrated high in vivo kinetic stability as a chelator for ${ }^{64} \mathrm{Cu}^{2+}$ ions [30], and our in vitro challenge experiments for ${ }^{64} \mathrm{Cu}$-NOTA-MAb B43.13 in the presence of an excess of purified SOD showed negligible trans-chelation (Additional file 1: Figure S10).

In contrast to its favorable in vitro targeting properties and proposed advantages, the ${ }^{64} \mathrm{Cu}-\mathrm{NOTA}-\mathrm{scFv}-$ B43.13 was less efficient in its role as a radiotracer for in vivo targeting of CA125. These observations are supported by other reports from in vivo use of such monomeric antibody fragments $[31,32]$. Although the $\mathrm{scFv}$ is expected to be renally cleared, we observed maximum renal trapping of the radioimmunoconjugate as early as $1 \mathrm{~h}$ p.i. (Additional file 1: Figure S11). Such a first pass effect precludes the scFv's bioavailability for any substantial tumor accumulation at later time points, thus accounting for the lower tumor uptake observed with this vector. This has prompted us to engineer the $\mathrm{scFv}$ into a diabody to achieve competitive avidity and better in vivo performance.
Ex vivo analysis by digital autoradiography reflected successful in vivo tumor accumulation of the ${ }^{64} \mathrm{Cu}$ NOTA-MAb-B43.13, while immunohistochemistry provided a panoramic view of CA125 expression in the tumor, indicating regions of high vascularity and stromal components in the tumor architecture. The lower half of the tumor was relatively rich in vasculature and formed suitable sites for neoplastic growth that appeared as densely stained tumor foci. Such vasculature also serves as a route of entry for targeted radioimmunoconjugates into the tumor. Most of the correlating signals from results between the three ex vivo techniques described in this study seem localized with strong CA125 staining in areas of dense vascular perfusion. This phenomenon may be a result of the limited penetration of antibody radioimmunoconjugates extravasating across blood vessels that constitute the aberrant tumor vasculature. This is exacerbated by the tumor's interstitial fluid pressure that can further impede the transport of antibodies once they are within the tumor along their pursuit to reach target cells that express CA125 [33-35]. Furthermore, 
the shedding properties of CA125 may create reservoirs of shed antigen within regions of high CA125 expression surrounding the tumor vasculature. This may lead to most of the radiotracer forming high molecular weight immunocomplexes at such junctions that may eventually be cleared via lymphatics.

Although the use of subcutaneous xenograft models of ovarian cancer allowed for easy assessment of tumor burden and an evaluation of the extent of tumor targeting, this advantage also contributes to being a limitation of this study. The next phase of testing this strategy will involve the use of alternative xenograft and/or transgenic animal models that more closely represent human EOC within the natural setting of the peritoneum, accompanied with an inducible recurrence of the disease. However, a drawback to using such a model would be the signal observed from organs of clearance such as the liver and kidneys that may conceal ovarian tumors, closer to these organs in the peritoneal cavity.

A concurrence of in vitro, in vivo, and ex vivo data taken from this preclinical proof-of-concept study represents an initial step towards investigating CA125 as a suitable target for the in vivo imaging of EOC. Drawing information from the present study and prior literature reports [12,36-38], it can be speculated that there may be a pathophysiological time interval between the overexpression of CA125 antigen on the surface of ovarian neoplasms and its subsequent shedding into the bloodstream of subjects at high risk for EOC and in recurrence. This time interval could be an existing gap between active small volume disease brewing to relapse out of the peritoneum and the limitations of presentday methods for its detection via CA125 immunoassays. Molecular imaging by immuno-PET may possibly bridge this gap to achieve a timely detection of EOC for better disease management.

\section{Conclusions}

In the work at hand, we have described the synthesis and preclinical radiopharmacological evaluation of ${ }^{64} \mathrm{Cu}$ labeled MAb-B43.13 and its derivative scFv for the PET imaging of CA125 in EOC. This approach offers both an opportunity for early detection of EOC in patients at high risk and a method to monitor patients for recurrence, by providing a non-invasive in vivo assessment of CA125 status. A robust model system was developed for preclinical testing, and antibody-based CA125-targeting radioimmunoconjugates were successfully produced with excellent immunoreactivity. Overall, our findings suggest that the more efficient tumor-targeting capabilities and favorable radiopharmacological profiles of the full-length antibody make it a suitable radiotracer for non-invasive in vivo evaluation of CA125 in EOC.

\section{Additional file}

Additional file 1: Supporting information. A file containing

supplementary table and figures.

\section{Abbreviations}

\%ID/g: percentage of injected dose per gram tissue; CA125: cancer antigen 125; EOC: epithelial ovarian cancer; MAP: maximum a priori; PET: positron emission tomography; ROI: region of interest, reversed phase; SEM: standard error of means; SUV: standardized uptake value; TAC: time-activity curve.

\section{Competing interests}

The authors declare that they have no competing interests.

\section{Authors' contributions}

SS was responsible for the synthesis and (radio) pharmacological evaluation of the immunoconjugates. He was responsible for writing the manuscript. MeW was responsible for animal experiments, including analysis of the PET data. MoW was supporting all radiopharmacological experiments. DG supported ex vivo analyses and immunofluorescence and immunohistochemistry experiments from tumor sections. BA supported cell culture of OVCAR3 and SKOV3 cells. SEL supported radiolabeling experiments. FW was responsible for the design of the study and critically revised the manuscript. All authors read and approved the final manuscript.

\section{Acknowledgements}

The authors would like to thank Mavanur R Suresh and Madi Madiyalakan at Quest Pharma Tech for providing the CA125-targeting vector platform; Razmik Mirzayans, Xuejun Sun, and Geralidine Barron for invaluable support with cell imaging; Jody Groenendyk for help with surface plasmon resonance; Vincent Bouvet for support with ${ }^{64} \mathrm{Cu}$ shipments and lab infrastructure; Jack Moore for mass spectrometry; and John Wilson, David Clendening, and Blake Lazurko from the Edmonton PET Center for excellent technical support.

\section{Author details}

${ }^{1}$ Faculty of Pharmacy and Pharmaceutical Sciences, University of Alberta, 8613 - 114 Street, Edmonton, AB T6G 2H1, Canada. ²Department of Oncology, University of Alberta, 11560 University Avenue, Edmonton, AB T6G 1Z2, Canada. ${ }^{3}$ Mallinckrodt Institute of Radiology, Washington University, 510 South Kingshighway Boulevard, Campus Box 8225, St. Louis, MO 63110, USA.

Received: 2 September 2014 Accepted: 24 October 2014

Published online: 12 November 2014

\section{References}

1. Howlader N, Noone AM, Krapcho M, Garshell J, Miller D, Altekruse SF, Kosary CL, Yu M, Ruhl J, Tatalovich Z, Mariotto A, Lewis DR, Chen HS, Feuer EJ, Cronin KA (Eds): SEER Cancer Statistics Review, 1975-2011. Bethesda, MD: National Cancer Institute. http://seer.cancer.gov/csr/1975_2011, based on November 2013 SEER data submission, posted to the SEER web site, April 2014.

2. Davis HM, Zurawski VR Jr, Bast RC Jr, Klug TL: Characterization of the CA 125 antigen associated with human epithelial ovarian carcinomas. Cancer Res 1986, 46(12 Pt 1):6143-6148.

3. Bast RC Jr, Xu FJ, Yu YH, Barnhill S, Zhang Z, Mills GB: CA 125: the past and the future. Int J Biol Markers 1998, 13:179-187.

4. Scholler N, Urban N: CA125 in ovarian cancer. Biomark Med 2007, 1:513-523.

5. Pignata S, Cannella L, Leopardo D, Bruni GS, Facchini G, Pisano C: Follow-up with CA125 after primary therapy of advanced ovarian cancer: in favor of continuing to prescribe CA125 during follow-up. Ann Oncol 2011, 22(Suppl 8):viii40-viii44.

6. Marcus CS, Maxwell GL, Darcy KM, Hamilton CA, McGuire WP: Current approaches and challenges in managing and monitoring treatment response in ovarian cancer. J Cancer 2014, 5:25-30.

7. Markman M: The role of CA-125 in the management of ovarian cancer. Oncologist 1997, 2:6-9.

8. Son H, Khan SM, Rahaman J, Cameron KL, Prasad-Hayes M, Chuang L, Machac J, Heiba S, Kostakoglu L: Role of FDG PET/CT in staging of recurrent ovarian cancer. Radiographics 2011, 31:569-583. 
9. Murakami M, Miyamoto T, lida T, Tsukada H, Watanabe M, Shida M, Maeda H, Nasu S, Yasuda S, Yasuda M, Ide M: Whole-body positron emission tomography and tumor marker CA125 for detection of recurrence in epithelial ovarian cancer. Int J Gynecol Cancer 2006, 16(Suppl 1):99-107.

10. Zimny M, Siggelkow W, Schröder W, Nowak B, Biemann S, Rath W, Buell U: 2-[Fluorine-18]-fluoro-2-deoxy-d-glucose positron emission tomography in the diagnosis of recurrent ovarian cancer. Gynecol Oncol 2001, 83:310-315.

11. Gadducci A, Cosio S: Surveillance of patients after initial treatment of ovarian cancer. Crit Rev Oncol Hematol 2009, 71:43-52.

12. Hori SS, Gambhir SS: Mathematical model identifies blood biomarker-based early cancer detection strategies and limitations. Sci Transl Med 2011, 3:109ra116.

13. Baum RP, Noujaim AA, Nanci A, Moebus V, Hertel A, Niesen A, Donnerstag B, Sykes T, Boniface G, Hör G: Clinical course of ovarian cancer patients under repeated stimulation of HAMA using MAb OC125 and B43.13. Hybridoma 1993, 12:583-589.

14. Schultes BC, Zhang C, Xue LY, Noujaim AA, Madiyalakan R: Immunotherapy of human ovarian carcinoma with OVAREX MAb-B43.13 in a human-PBL-SCID/BG mouse model. Hybridoma 1999, 18:47-55.

15. Berek JS: Immunotherapy of ovarian cancer with antibodies: a focus on oregovomab. Expert Opin Biol Ther 2004, 4:1159-1165.

16. WU AM: Antibodies and antimatter: the resurgence of immuno-PET. J Nucl Med 2009, 50:2-5.

17. Holland JP, Divilov V, Bander NH, Smith-Jones PM, Larson SM, Lewis JS: ${ }^{89} \mathrm{Zr}$-DFO-J591 for immunoPET of prostate-specific membrane antigen expression in vivo. J Nucl Med 2010, 51:1293-1300.

18. Viola-Villegas NT, Rice SL, Carlin S, Wu X, Evans MJ, Sevak KK, Drobjnak M, Ragupathi G, Sawada R, Scholz WW, Livingston PO, Lewis JS: Applying PET to broaden the diagnostic utility of the clinically validated CA19.9 serum biomarker for oncology. J NuCl Med 2013, 54:1876-1882.

19. Noujaim AA, Baum RP, Sykes TR, Sykes CJ, Hertel A, Niesen A, Donnerstag B, Boniface G, Hör G: Monoclonal antibody B43.13 for immunoscintigraphy and immunotherapy of ovarian cancer. In Current Tumor Diagnosis: Applications, Clinical Relevance, Trends. Edited by Munich KR. : Zuckschwerdt Verlag; 1994:823-829.

20. Kriangkum J, Xu B, Gervais C, Paquette D, Jacobs FA, Martin L, Suresh MR: Development and characterization of a bi-specific single chain antibody against T cells and ovarian carcinoma. Hybridoma 2000, 19:33-41.

21. Wang WW, Das D, McQuarrie SA, Suresh MR: Design of a bifunctional fusion protein for ovarian cancer drug delivery: single-chain anti-CA125 core-streptavidin fusion protein. Eur J Pharm Biopharm 2007, 65:398-405.

22. Cooper MS, Ma MT, Sunasee K, Shaw KP, Williams JD, Paul RL, Donnelly PS, Blower PJ: Comparison of ${ }^{64} \mathrm{Cu}$-complexing bifunctional chelators for radioimmuno-conjugation: labeling efficiency, specific activity, and in vitro/in vivo stability. Bioconjug Chem 2012, 23:1029-1039.

23. Kume M, Carey PC, Gaehle G, Madrid E, Voller T, Margenau W, Welch MJ, Lapi SE: A semi-automated system for the routine production of copper-64. Appl Radiat Isot 2012, 70:1803-1806.

24. Lindmo T, Boven E, Cuttitta F, Fedorko J, Bunn PA Jr: Determination of the immunoreactive fraction of radiolabeled monoclonal antibodies by linear extrapolation to binding at infinite antigen excess. $J$ Immunol Methods 1984, 72:77-89.

25. Prat J: New insights into ovarian cancer pathology. Ann Oncol 2012, 23(Suppl 10):x111-x117.

26. McQuarrie SA, Baum RP, Niesen A, Madiyalakan R, Korz W, Sykes TR, Sykes CJ, Hör G, McEwan AJ, Noujaim AA: Pharmacokinetics and radiation dosimetry of ${ }^{99 \mathrm{~m}} \mathrm{Tc}$ labeled monoclonal antibody B43.13 in ovarian cancer patients. Nucl Med Commun 1997, 18:878-886.

27. Lutz AM, Ray P, Willmann JK, Drescher C, Gambhir SS: 2-Deoxy-2-[F-18] fluoro-D-glucose accumulation in ovarian carcinoma cell lines. Mol Imaging Biol 2007, 9:260-266

28. Xiao Z, McQuarrie SA, Suresh MR, Mercer JR, Gupta S, Miller GG: A threestep strategy for targeting drug carriers to human ovarian carcinoma cells in vitro. J Biotechnol 2002, 94:171-184.

29. Fang J, Nakamura H, Maeda H: The EPR effect: unique features of tumor blood vessels for drug delivery, factors involved, and limitations and augmentation of the effect. Adv Drug Deliv Rev 2011, 63:136-151.

30. Dearling JL, Voss SD, Dunning P, Snay E, Fahey F, Smith SV, Huston JS, Meares CF, Treves ST, Packard AB: Imaging cancer using PET - the effect of the bifunctional chelator on the biodistribution of a ${ }^{64} \mathrm{Cu}$-labeled antibody. Nucl Med Biol 2011, 38:29-38.
31. Wittrup KD, Thurber GM, Schmidt MM, Rhoden JJ: Practical theoretic guidance for the design of tumor-targeting agents. Methods Enzymol 2012, 503:255-268.

32. Schneider DW, Heitner T, Alicke B, Light DR, McLean K, Satozawa N, Parry G, Yoo J, Lewis JS, Parry R: In vivo biodistribution, PET imaging, and tumor accumulation of ${ }^{86} \mathrm{Y}$ - and ${ }^{111} \mathrm{In}$-antimindin/RG-1, engineered antibody fragments in LNCaP tumor-bearing nude mice. J NuCl Med 2009, 50:435-443.

33. Thurber GM, Schmidt MM, Wittrup KD: Factors determining antibody distribution in tumors. Trends Pharmacol Sci 2008, 29:57-61.

34. Jain RK: Transport of molecules, particles, and cells in solid tumors. Annu Rev Biomed Eng 1999, 1:241-263.

35. Heldin $\mathrm{CH}$, Rubin K, Pietras K, Ostman A: High interstitial fluid pressure - an obstacle in cancer therapy. Nat Rev Cancer 2004, 4:806-813.

36. McQuarrie SA, Riauka T, Baum RP, Sykes TR, Noujaim AA, Boniface G, MacLean GD, McEwan AJ: The effects of circulating antigen on the pharmacokinetics and radioimmunoscintigraphic properties of ${ }^{99 m} \mathrm{Tc}$ monoclonal antibodies in cancer patients. J Pharm Pharm Sci 1998, 1:115-125.

37. Masuho Y, Zalutsky M, Knapp RC, Bast RC Jr: Interaction of monoclonal antibodies with cell surface antigens of human ovarian carcinomas. Cancer Res 1984, 44:2813-2819.

38. Marth C, Zeimet AG, Windschwendter M, Daxenbichler G: Regulation of CA 125 expression in cultured human carcinoma cells. Int J Biol Markers 1998, 13:207-209.

\section{doi:10.1186/s13550-014-0060-4}

Cite this article as: Sharma et al:: Immuno-PET of epithelial ovarian cancer: harnessing the potential of CA125 for non-invasive imaging. EJNMMI Research 2014 4:60.

\section{Submit your manuscript to a SpringerOpen ${ }^{\circ}$ journal and benefit from:}

- Convenient online submission

- Rigorous peer review

- Immediate publication on acceptance

- Open access: articles freely available online

- High visibility within the field

- Retaining the copyright to your article

Submit your next manuscript at $>$ springeropen.com 